\title{
Human Identification Using Foot Features
}

\author{
Prof.Dr. Kadhim M.Hashem, Fatima Ghali \\ ${ }^{a}$ Department of computer science University of Thi-Qar, Thi-Qar, Iraq
}

\begin{abstract}
The goal of this paper is to investigate a new technique for human identification using foot features. This work can be mainly decomposed into image preprocessing, feature extraction and pattern recognition by Artificial Neural Network (ANN).

Foot images are rarely of perfect quality. To obtain good minutiae extraction in foot with varying quality, we conducted preprocessing in form of image enhancement and binarization .To extract features from human foot based on shape geometry of foot boundaries by extracting 16 geometric features from a human foot image. The foot center has been determined, and then the distances between the center point and outer points are measured with different angles. The angles are from $30^{\circ}$ to $360^{\circ}$ by increment with $30^{\circ}$ gradual. The 13 th feature that can be extracted is the length of a foot which is defined as the distance between the top point of the foot and the bottom point. The 14th, 15th and 16th are three major features the width of the foot. The first width is passing through center point, therefore, the second widths of foot is measured from the upper part above the center point and third width from the region the center point under the center point at the bottom of the foot. Euclidean distance is used in the proposed system. Artificial Neural network used for recognition.

MATLAB version 8.1(R2013a) and windows 7 with 32 bit is used to build the application and performed on pc of core i3 processor, and our test system on 40 persons, results were satisfactory up to more $92.5 \%$.
\end{abstract}

Index Terms: Pattern Recognition, Foot Geometry, Euclidean Distance, image processing.

(C) 2016 Published by MECS Publisher. Selection and/or peer review under responsibility of the Research Association of Modern Education and Computer Science.

\section{Introduction}

In a Growing digital world, credible personal authentication has become an important human computer interface activity. National security, e-commerce, and access to computer networks are some examples where establishing a person's identity is vital. Most existing security measures depend on knowledge-based approach as passwords or token-based approach like swipe cards and passports to control access to physical and virtual spaces. However, all over the world, such methods are not very secure. Token cards based access may be

* Corresponding author.

E-mail address: Kadhimmehdi63@gmail.com, Fatimaghali33@gmail.com 
shared or stolen. Passwords and PIN (Personal Identification numbers) may be stolen electronically. Furthermore, they cannot differentiate between an authorized user and a person having access to tokens or knowledge. Biometrics such as fingerprint, face and foot offers reliable personal authentication that can address these problems and is gaining both public and government acceptance [1].

Biometrics are the study of an automated method to recognize a human being based on his behavioral or physical characteristics. The behavior characteristics are such as signature, gait, and voice...etc. while physical characteristics refer to the structural pattern of human such as fingerprint, foot, ear, face, and hand geometry...etc. [2] [3].

Biometric systems can be divided into two categories (Verification systems and Identification systems). Verification systems say "are you the one who you claim to be?" and Identification systems say "who you are?" Verification mode: In the verification mode, the system deals with the identity of the person by comparing the captured biometric data with her special biometric template(s) stored in the database system. In this system, the person who desires to be recognized claims an identity, usually by PIN( Personal Identification number), a user name, a smart card, etc., and the system conducts a one-to-one comparison to determine whether the claim is true or not (e.g., "Does this biometric data belong to Mustafa?").Verification of Identity is usually used for positive recognition, where the goal is to prevent multiple people from using the same identity. Identification mode: In identification mode, the system recognizes a person by searching the templates for all users in the database for a match. Therefore, the system conducts a one-to-many comparison to establish a person identity (or fails if the subject is not registered in the system database) without the subject having to claim an identity (e.g., "Whose biometric data is this?"). Identification is a critical element in negative recognition applications where the system identifies whether the person is who she (implicitly or explicitly) denies to be. The purpose of negative recognition is to prevent a single individual from using multiple identities. Identification can also be used in positive recognition for convenience (the user is not required to claim an identity) [4].

In this paper a new approach is presented for personal identification using foot-biometric based on shape geometry and recognition using artificial neural networks (ANN).

\section{Related works}

In 2005 T. Kurgan, A. Yamaguchi and S. Furukawa presented A Method to Measure Foot Print Similarity for Gait Analysis. This Provide a novel approach based on gait and footprint analysis. Health care providers in Japan assess the recovery status of patients by detecting a variation in the patient's style of walking. In the first phase of psychoanalysis, the manner of walking is uneven. By way of rehabilitation progresses, the mode of walking of the patient turn into stable state. Consequently, changes in the footprint images of the patients may be identified. The techniques of binarization of a foot print image, noise-reduction, and damage and stretching to smoothening of the edge of the binary image to discover the edge of the footprint image are defined. In addition discrete Fourier transformation has been applied to measure the correspondence of footprint patterns and inverse discrete Fourier transformations to eliminate the high frequency mechanisms in the footprint pattern are described [5].

In 2008 Andreas Uhl and Peter Wild presented Footprint-based biometric verification. It explore an approach with the characteristics of biometric foot which is established through geometry, shape and the texture. The Image enhancement and the feature extraction stages highlighting on definite characteristics of the foot geometry, their durability and the uniqueness properties, respectively, are also explained by them. Collectability and universality are the important issues which are also reflected. The Gait Recognition is a remarkable signal processing tool for the biometric proof of identity [6].

In 2012 V. Kumar and M.Ramakrishnan presented Footprint Recognition with COP Using Principle Component Analysis (PCA). In their research, the center of pressure (COP) has been taken out by means of stationary technique from the resized attuned footprint image. COP value is determined using the pixel coordinates, these values are further trained using Principle Component Analysis (PCA), and then stored in templates. PCA diminishes the great dimensionality of the data space to the smaller fundamental 
dimensionality of feature space, then and there the Matching algorithm associates the test data based upon the three features using the template and demonstrates whether the user is legitimate or not. From the above discussion, it is very clear that for personal footprint based identification there are only two domains available: (i) Gait based system, and (ii) Minutia based system. Additionally, one can use any of the hybrid direction i.e. Gait together with Minutia based on personal identification [7].

\section{Growth of the Human Foot}

The foot of the new born child has only one bone. The rest of the foot is mainly cartilage. Not all the bones of the foot are present during the periods of newly born and in infancy. The final primary and secondary bony structures would have gradually emerged by puberty. When a child reaches 3 years of age a lot of the cartilage would have become bones and by the age of 6 all the bones would have taken shape but would still be partially consisting of cartilage.

The growth of the human foot comes gradual in stages. Studies show that during the first ten years of a child's life the foot grows nearly one-half of an inch a year. Largely the average yearly growth slows down between the ages of 10 and 20 years, with maturity of growth to be between the ages of 19 and 20 years [8].

\section{Foot Geometry}

The foot geometry technology is based on the fact that adult person has a special shape of foot which belongs to them only, it is not affected by factors of time and aging. The foot size is relatively stable if the human has completed the growth phase and not properly use this type of measurement on children or those who are in the growth phase of their life. In other words, adult persons owning a distinctive unique foot among the rest of the people, foot geometry system always bases on the biometric features of the foot and some specific measurements such as length and width of the foot [9].

\section{The proposed foot recognition system}

Human identification using foot image is one of the biometric techniques. Foot recognition system can be divided into four major steps (image Acquisition, image preprocessing, features extraction and recognition using MLP). Figure (1) shows the steps for the foot recognition system.

\section{A. Image Acquisition}

The investigate the same conditions on the all acquitted images, the stand base has been used. The distance between camera stand and human foot is fixed ( 2 meters). The lighting and background is fixed for images. Camera's resolution equals 20 Mega pixel, and with zoom equals 50 degrees. The images are taken for right foot only for all person.

\section{B. Image preprocessing}

At first it's necessary to apply several pre-processing steps on the original foot image to produce images with same condition to be used in features extraction procedure. In this step, the foot is processed prior to feature extraction in order to eliminate noise and to enhance the features which are used in the next steps, such as gray scale conversion, image Resize, image Enhancement, binarization, edge extraction in an image and morphological operations. The edge detection is done using the well-known canny edge detector [10]. The gray value array with size $144 \times 96$ is used as input to canny detector, the output a binary image the threshold must be selected carefully to ensure the data information preserved after the binarization [11]. The threshold values used in this paper were selected by equation: 
Threshold $=($ mean $($ grayscale image $) * 0.001) / 2$

Figure (2) shows a grayscale image and its corresponding edge-detected binary image obtained from canny edge detector.

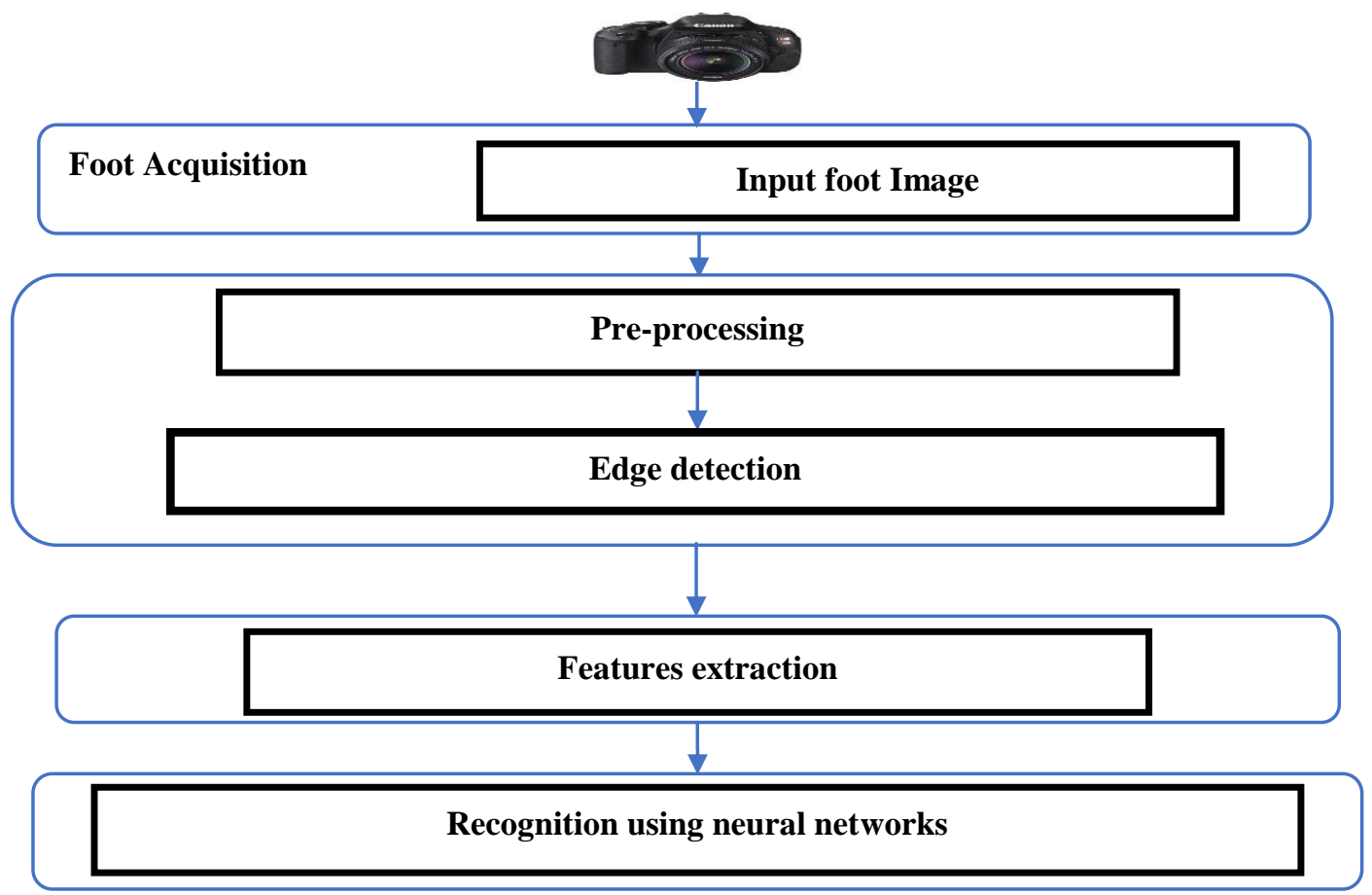

Fig.1. Main Block diagram of the proposed approach
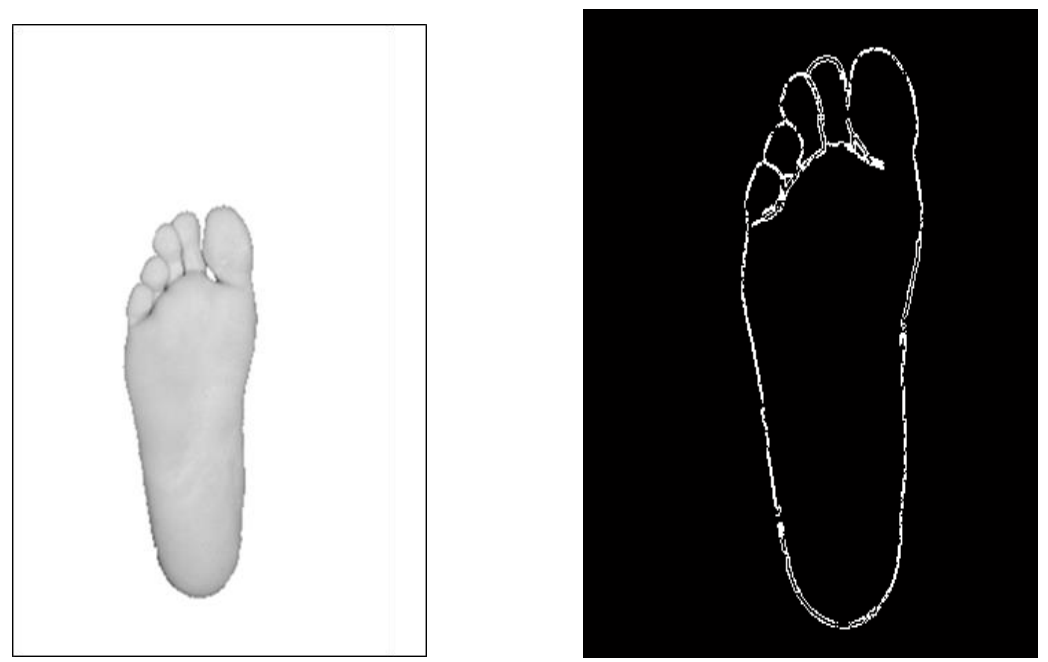

Fig.2. Grayscale image and its corresponding edge detected binary image 


\section{Features extraction:}

There are several features that can be extracted from the geometry of the foot. This paper presents a new approach for extraction features from human foot based the on shape geometry of its boundary frame. This could be achieved by extracting 16 geometric features from a human foot, and by determining the foot center and then extracting 12 geometric features. By calculating the distance between the center and outer point by different angles, the angles are from $30^{\circ}$ to $360^{\circ}$ increased by $30^{\circ}$ gradual.

The 13th feature that can be extracted is the length of a foot which is defined as the distance between the top point of the foot and the bottom points. The 14th, 15th and 16th have three major features as the width of the foot. The center of the foot can also be used to measure of foot one width, therefore, we can find two widths of foot from the upper part above the center point and third width we found under the center point at the bottom of the foot. The Foot length is defined as the distance between the top point and the bottom foot point. The first step is to determine the top and bottom position of each foot. The length of the foot is then calculated by taking the distance between the top and bottom points, as shown in figure (3)
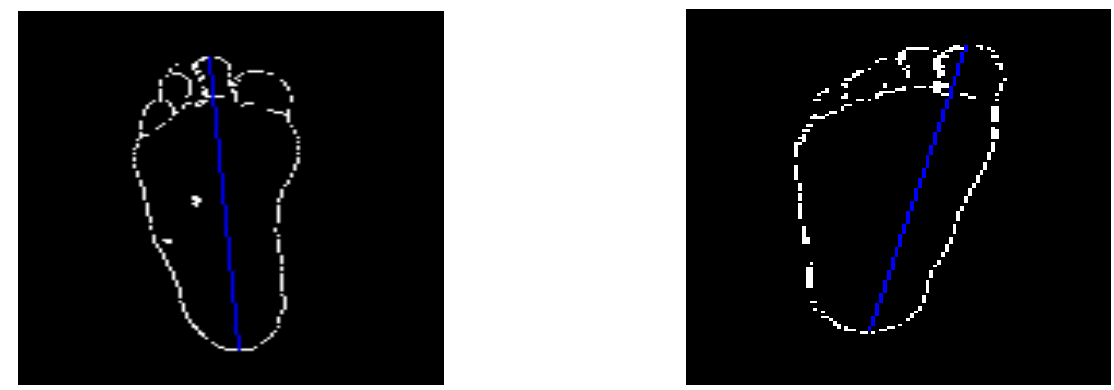

Fig.3. The length foot

The center foot is defined by four points, first point at the top of the foot, second point at the bottom of the foot, third point is the right of the foot and the fourth point is the left of the foot. Through these four points, we can determine foot center, as shown in figure (4). After determine the foot center, then calculate the distance between the center and outer points, measured that from different angles. The angles are from $30^{\circ}$ to $360^{\circ}$ increment by $30^{\circ}$ gradual, as shown in figure (5).
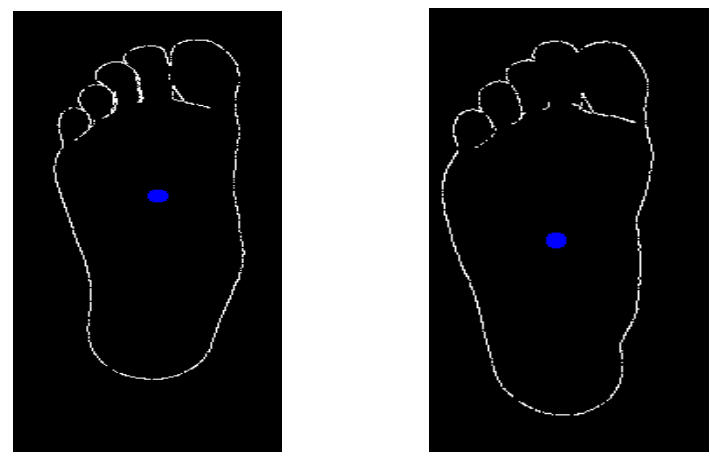

Fig.4. The center of the foot 

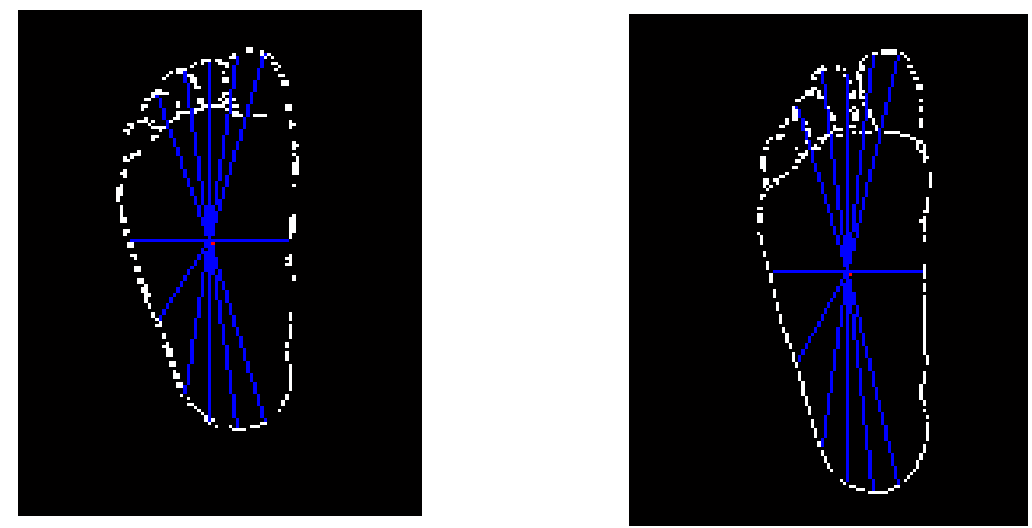

Fig.5. The distance between the center and outer point by different angles were measured.

The final features foot widths. The Foot width is defined as the distance between the left point and the right point. In the proposed system, we can find three widths for the foot. The first width can be found from center point, second width can be found in the upper part above the center point and third width can be found at the bottom under the center point, as shown in figure (6).
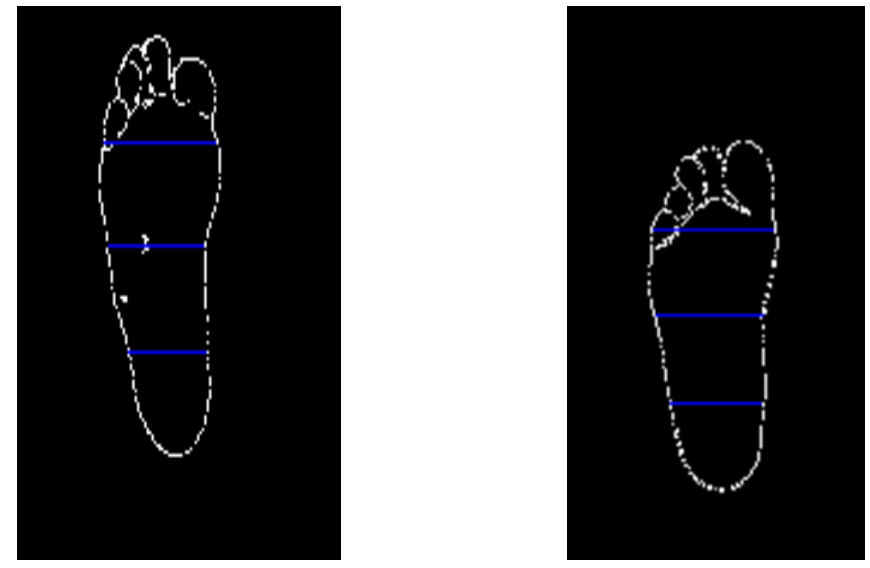

Fig.6. The three width in the foot

\section{Recognition using Artificial neural networks:}

The designed ANN which is used in process consists of three layers: Input layer, one hidden layer and output layer, the activation function was selected for the hidden and output layers [12] [13]. After features extraction, we divided the samples into two groups. The first group is used to learn ANN (training phase). The second group is used to calculate or test the efficiency of the ANN to distinguish different samples from that used in the learning phase (testing phase).

The number of nodes in the input layer depends on the number of features extracted from each sample. In this study, the number of nodes in the input layer=16. For this specific problem, the number of output neurons equals the number of bits that represent the number of persons. In this study, the number of nodes in the output layer $=6$ because the number of persons is 40 .The number of hidden layer and the number of nodes in the hidden layer in this study is 11 . As shown in figure (7). 


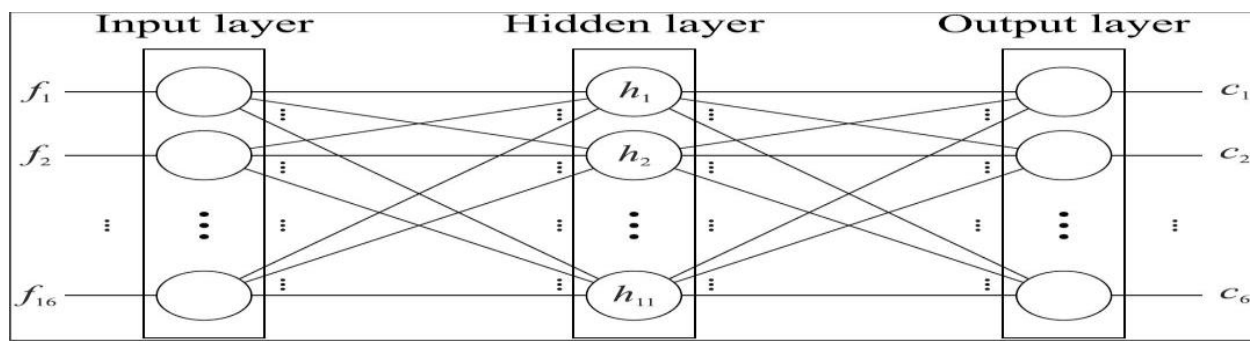

Fig.7. Multi-layered Perceptron neural network architecture

\section{Results}

The number of foot images used in training are 120 images, the number of images used in the test is 40 images.

\section{A. Training Results}

The initial parameters of the neural networks used in these tests are given below:

Type: Feed forward back propagation network-

Number of layers: 3 (input, one hidden, output layer)

- Number of neurons in input layer: Number of features extraction from foot $=16$

- $\quad$ Number of neurons in hidden layer: 11

- Number of neurons in output layer: 6

- Function of the layer: sigmoid function

- Number of epochs used in training: 100

- $\quad$ Backprop weight: [0.4,-0.4]

- Bias learning: 1

For the most part, a network is trained by changing the weights of the connections between nodes [14] .These weights can be randomly chosen or individually chosen. A program randomly generates values for connection weights [15]. Then, the network is given an input, and it is allowed to process the information through its nodes to produce an output. System is trained many times to give the better recognition rate as shown in Figure (8). Figure (9) shows the recognition rate is 100 and mean square error (mse) starts decreasing gradually.

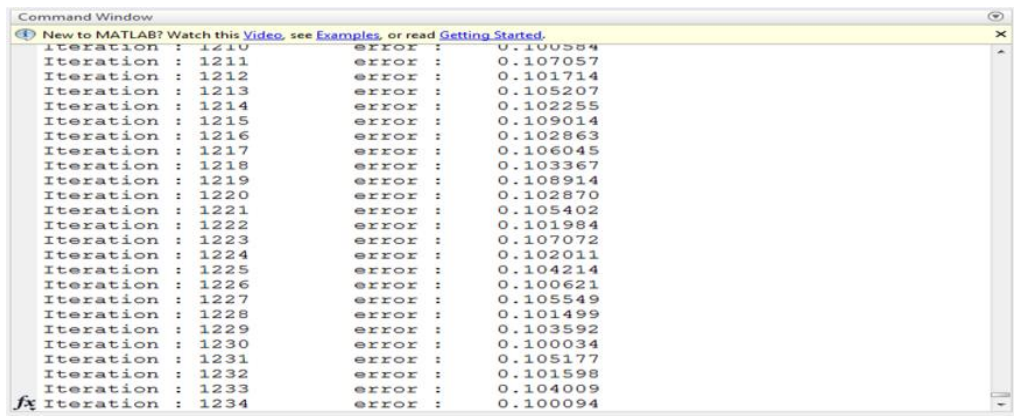

Fig.8. Training by neural network 


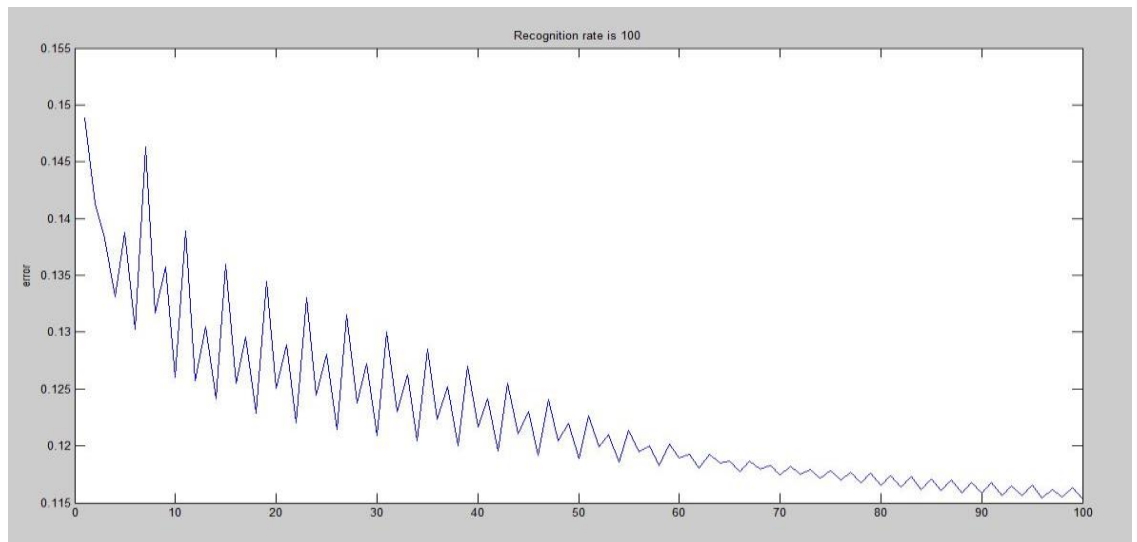

Fig.9. Shows mean square error (mse)

\section{B. Testing Results}

The testing results of the foot recognition system are obtained using MLP, the images are taken in different times for the same persons. Performed the testing in three different experiments:

In first experiment, we selected 40 images of 40 persons. Recognition rate was $92.5 \%$.

In the second experiment, we selected 31 images randomly for 31 persons. Recognition rate is $93.5 \%$.

In the third experiment, we selected 21 images randomly for 21 persons. Recognition rate is 95.2. (As shown in figure (10)).Table (1) shows the recognition rate of the three experiments.

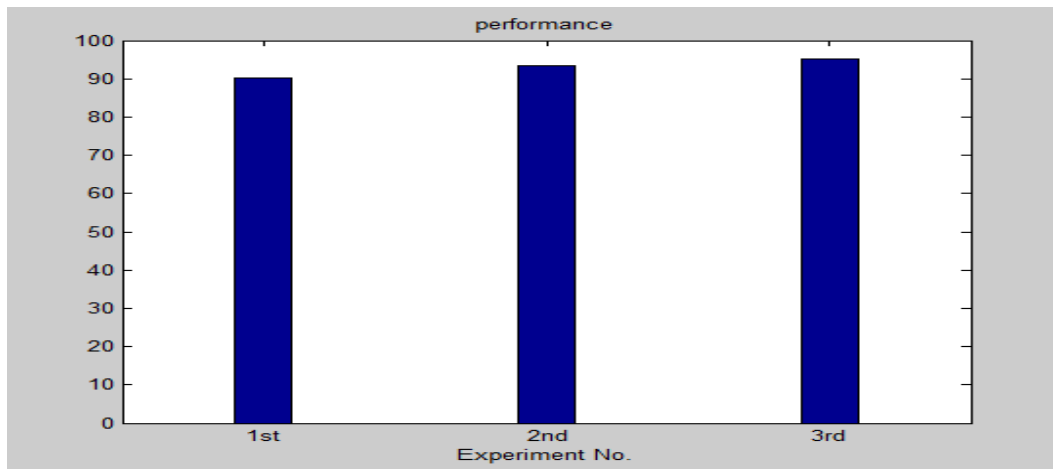

Fig.10. Shows performance in testing

Table 1. Recognition rates of the system for foot recognition tested images

\begin{tabular}{|l|l|l|}
\hline Foot in database & Image was recognized & Recognition rate \\
\hline 40 & $\mathbf{3 7}$ & $\mathbf{9 2 . 5 \%}$ \\
\hline 31 & $\mathbf{2 9}$ & $\mathbf{9 3 . 5 \%}$ \\
\hline 21 & $\mathbf{2 0}$ & $\mathbf{9 5 . 2 \%}$ \\
\hline
\end{tabular}




\section{Conclusions}

1. Using foot to identify or verify an individual's identity

2. The use of artificial neural networks is better than traditional methods and give results acceptable in pattern recognition. Artificial neural network is an intelligent way and more effective compared to other traditional ways for adoption on the training process where this method is the most appropriate solution methods

\section{References}

[1] K.K.Nagwanshi and S. Dubey," Biometric Authentication using Human Footprint", International Journal of Applied Information Systems, Foundation of Computer Science, New York, USA,vol.3, No.7, August ,2012.

[2] K. V. Singh," Application of Neural Network in Fingerprint Identification", Thapar University, Computer science and engineering department, M.ScThesis, July, 2010.

[3] S. Ashish," Design of a Hand Geometry Based Recognition System", Indian Institute of Technology Kanpur, Department of Computer Science \& Engineering,M.Sc.Thesis, Jan, 2007.

[4] S.A. Sahmoud," Enhancing Iris Recognition", Islamic University, Computer Engineering Department, M. Sc.Thesis, 2011.

[5] T. Kuragano, A. Yamaguchi and S. Furukawa, "A Method to Measure Foot Print Similarity for Gait Analysis," in IEEE-Computer Society- 2005 International Conference on Computational Intelligence for Modelling, Control and Automation, and International Conference Intelligent Agents, Web Technologies and Internet Commerce (CIMCA-IAWIC'05), 2005.

[6] D.Naga Shailaja," A simple Geometric Approach for Aar Recognition", Engineering Indian institute of Technology, Kanpur, Department of computer science, M.Sc. Thesis June,2006.

[7] A. Uhl and P. Wild, "Footprint-based biometric verification," Journal of Electronic Imaging-Society of Photo-Optical Instrumentation Engineers, vol. 17, no. 1, p. 011016, Mar, 2008.

[8] V. Kumar and M.Ramakrishnan, "Footprint Recognition with COP Using Principle Component Analysis (PCA)," Journal of Computational Information Systems, vol. 8, no. 12, pp. 4939-4950, 2012.

[9] Ö .H. ÖZKAN," A research on Foot Twear and Foot Interaction ON Through Anatomy and Human Engineering", The Graduate School of Engineering and Sciences, Izmir Institute of Technology, M.Sc. Thesis, January, 2005.

[10] W. Wang, X. Ping and Y. Ding, "Footprint Heavy Pressure Surface Pick-up and Description," in IEEE Computer Society-Third International Conference on Image and Graphics (ICIG'04), Hong Kong, China, 18-20 December 2004 .

[11] T.A.Hussein,"Arabic-text extraction from video images", University of Basrah, college of science, Department of computer science, MS. Thesis, 2013.

[12] D. Kumar, S. P. Tripathi and D. Misra," A Review report on fingerprint image enhancement filter", International Journal of Computer Science Engineering and Information Technology Research, ISSN 2249-6831, Vol. 3, Issue 2, pp. 403-416, Jun 2013.

[13] K .Geetha and S. Santhosh Baboo," Systematic Study of Fuzzy, Neural Network and Neurofuzzy Systems", Journal of Engineering Research and Application Vol. 3, No. 5, pp.884-889, Oct, 2013.

[14] V. Sharma, S. Rai and A. Dev," A Comprehensive Study of Artificial Neural Networks", International Journal of Advanced Research in Computer Science and Software Engineering Vol., Issue 10, October, 2012. 
[15] P.Sibi, S.A.JONES and P.Siddarth," Analysis of Different Activation Functions Using Back Propagation Neural Networks", Journal of Theoretical and Applied Information Technology Vol. 47 No.3, January 2013.

\section{Authors' Profiles}

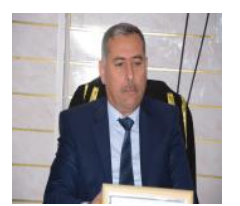

Kadhim M.Hashem received his BS degree in computer science from Al- Basra University, lraq, in 1985, received his MS degree in computer Science from Al- Technology University, lraq, in 1991, and received his PHD degree from Al- Basra University, lraq, in 2006.His interesting area in image processing, Biometric, Artificial intelligent, pattern recognition.

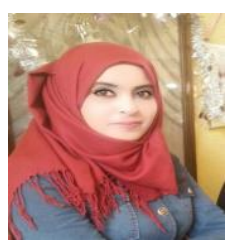

Fatima G. Dahal received her BS degree in computer science from University of Thiาר-Qar College of Education for pure science, lraq, in 2013, and currently MS student in computer science from University of Thiרา-Qar, her interesting area in Biometric, image processing, Artificial Neural Networks.

How to cite this paper: Kadhim M.Hashem, Fatima Ghali,"Human Identification Using Foot Features", International Journal of Engineering and Manufacturing(IJEM), Vol.6, No.4, pp.22-31, 2016.DOI: 10.5815/ijem.2016.04.03 